Background To assess the validity of the pit patterns Kudo classification and the NBI international colorectal endoscopic (NICE) classification on detection of the depth infiltration of colorectal laterally spreading tumours (LST).

Methods Colorectal laterally spreading tumours were collected from digestive endoscopy centre of PLA army general hospital from Mar. 2010 to May 2016. There were 250 LSTs were mucosal stained, grouped according to pit patterns Kudo classification; 208 LSTs were observed by NBI, grouped according to NICE classification. Then compared the pit patterns classification and NICE classification with pathologic diagnosis, separately.

Results The pit pattern of the most LST was type IIIL $(30.56 \%)$ and IV (32.94\%). V pit pattern which signifies the cancerated metaplasia has large proportion in granular-nodular mixed (LST-GM) and non-granular-flat-elevated (LST-NGF), $29.11 \%$ and $41.93 \%$ respectively. In the LST - GM, V1 accounted for 24.05\%, and In LST - NGPD $\mathrm{V}_{\mathrm{N}}$ accounted for $19.35 \%$, higher than other subtypes. The accuracy of type $\mathrm{V}$ for predicting submucosal invasion is $100 \%$, the accuracy of type $\mathrm{V}_{\mathrm{N}}$ for predicting colorectal early cancer is $100 \%$, for predicting submucosal invasion is $58.33 \%$. There were 101 LSTs with low-grade dysplasia; all the lesions were NICE2 type, 59 LSTs with high-grade dysplasia, 93.22\% (55/59) for NICE2 type, $6.78 \%$ (4/59) for NICE3 type. A total of 13 cases were submucosal carcinoma (SM-d), 61.54\% (8/13) for NICE2 type, 38.46\% (5/13) for NICE3 type.

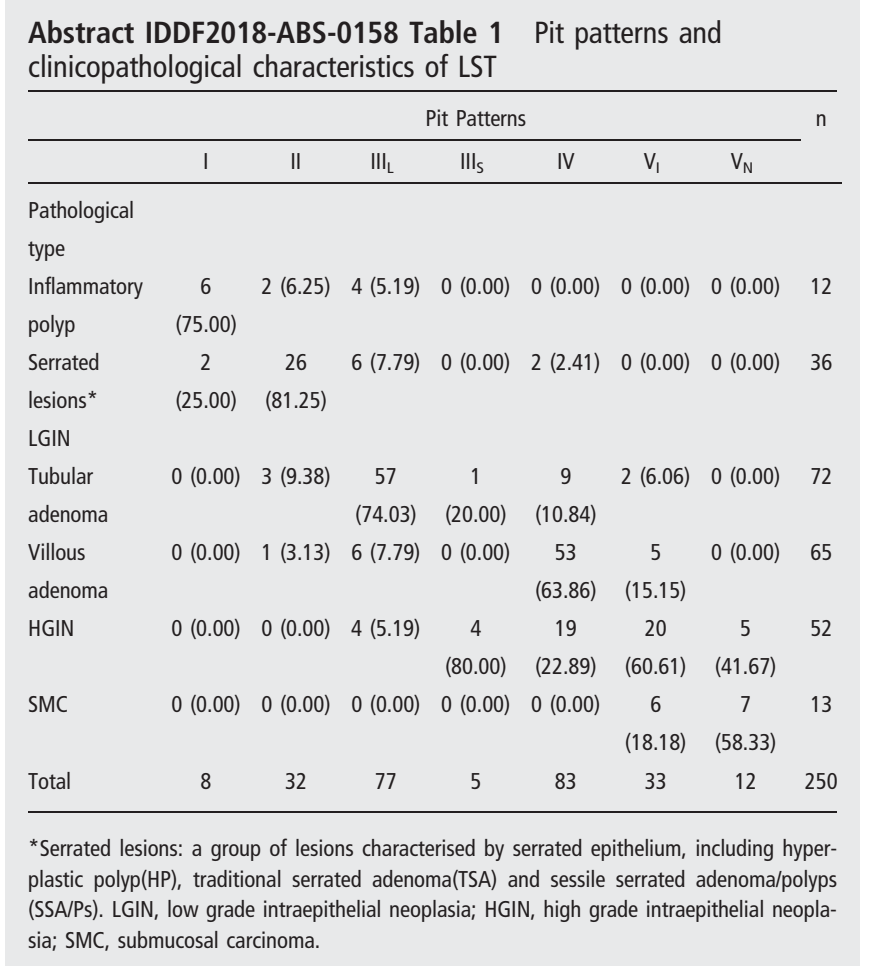

Conclusions In terms of LSTs, the type IIIL is the predominant pit pattern in LST-NG, whereas the type IV pit pattern occurs in the LST-G, the pit pattern V and NICE3 indicate the large possibility of submucosal invasion, should be noticed by endoscopic physicians.

\section{IDDF2018-ABS-0159 ENDOSCOPIC CHARACTERISTICS OF COLORECTAL LATERALLY SPREADING TUMOURS}

Xin Fan*. PLA Army General Hospital, China

\subsection{6/gutjnl-2018-IDDFabstracts. 133}

Background To analyse the endoscopic characteristics and therapeutic methods of colorectal laterally spreading tumours.

Methods Colorectal laterally spreading tumours were collected from digestive endoscopy centre of PLA army general hospital from Mar 2010 to May 2016. Their relationship between morphology? predilection site and histopathology were analysed retrospectively.

Results A total of 615 colorectal laterally spreading tumours (551patients) were collected. The average diameter of lesion was 22.2? $14.7 \mathrm{~mm}$, the size was from 10 to $20 \mathrm{~mm}$ in the most $(66.5 \%)$, and LSTs were predominant in the proximal colon (55.4\%). Granular-homogenous(LST-GH) was detected more frequently in the ileocecal region and ascending colon (75/140,53.7\%); Granular-nodular mixed (LST-GM) was detected more frequently in the rectum and sigmoid colon (91/140,65.0\%); Non-granular-flat-elevated (LST-NGF) and non-granular-pseudo-depressed (LST-NG-PD) were preferentially located in the proximal colon, especially more common in transverse colon, account for $25.9 \%$ and $32.1 \%$ respectively. Among lesions intramucosal carcinoma and submucosal carcinoma was $19.67 \%(121 / 615)$, submucosal carcinoma was $3.25 \%(20 / 615)$. LST-GM and LST-NGPD had a higher incidence of intramucosal carcinoma and submucosal carcinoma compared to the other subtypes, $43.28 \%$ and $34.52 \%$, respectively. LST-NGPD was highest rates of submucosal invasion: $14.29 \%$ ? LST-GM was predominant in the rectum; the mean tumour size was larger in the rectum than the colon (31.49? 23.09 vs 20.82 ?9.86,p<0.05), higher incidence of high-grade dysplasia and submucosal carcinoma $(36.43 \%$ vs $14.95 \%$, $\mathrm{p}<0.001)$.

\begin{tabular}{|c|c|c|c|c|}
\hline & $\begin{array}{l}\text { Colon, } \\
n=475\end{array}$ & $\begin{array}{c}\text { Rectum, } \\
\mathrm{n}=140\end{array}$ & $p$ value & All, $n=615$ \\
\hline Age, medium (range) & $61(17-92)$ & $64(31-88)$ & $<0.05$ & $62(17-92)$ \\
\hline Male gender & $281(59.16)$ & 78 (55.71) & 0.47 & $359(58.37)$ \\
\hline Subtypes of LST & & & $<0.05$ & \\
\hline LST-GH & $109(22.95)$ & 31 (22.14) & & $140(22.76)$ \\
\hline LST-GM & 78 (16.42) & $62(44.29)$ & & $140(22.76)$ \\
\hline LST-NGF & $216(45.47)$ & $35(25.00)$ & & $251(40.81)$ \\
\hline LST-NGD & $72(15.16)$ & $12(8.57)$ & & $84(13.66)$ \\
\hline Size, mean $\pm S D(m m)$ & $20.82 \pm 9.86$ & $31.49 \pm 23.09$ & $<0.05$ & $\begin{array}{c}23.25 \\
\pm 14.69\end{array}$ \\
\hline $\begin{array}{l}\text { Histological } \\
\text { classification }\end{array}$ & & & $<0.0001$ & \\
\hline Low-grade dysplasia & 275 (57.68) & $74(53.57)$ & & 349 (56.75) \\
\hline High-grade dysplasia & $58(12.21)$ & $44(31.43)$ & & $102(16.59)$ \\
\hline Submucosal cancer & $13(2.74)$ & $7(5.00)$ & & $20(3.25)$ \\
\hline
\end{tabular}


Conclusions LSTs were predominant in the proximal colon, but a greater proportion of LST-GM, greater mean-size, and greater presence of submucosal cancer found in rectal LST. LST-GM and LST-NGPD had a higher incidence of intramucosal carcinoma and submucosal carcinoma, in particular, LSTNGPD was highest rates of submucosal invasion, should be noticed by endoscopic physicians.

\section{IDDF2018-ABS-0160 ENDOSCOPIC CHARACTERISTICS, TREATMENT AND FOLLOW-UP OF COLORECTAL LATERALLY SPREADING TUMOURS}

Zilin Kan*, Peng Jin. PLA Army General Hospital, China

\subsection{6/gutjnl-2018-IDDFabstracts.134}

Background To analyse the endoscopic characteristics and therapeutic methods of colorectal laterally spreading tumours.

Methods Colorectal laterally spreading tumours were collected from digestive endoscopy centre of PLA army general hospital from Mar 2010 to May 2016. Their relationship between morphology, predilection site, pit patterns and histopathology were analysed retrospectively. And compared the follow-up results of different endoscopic treatment.

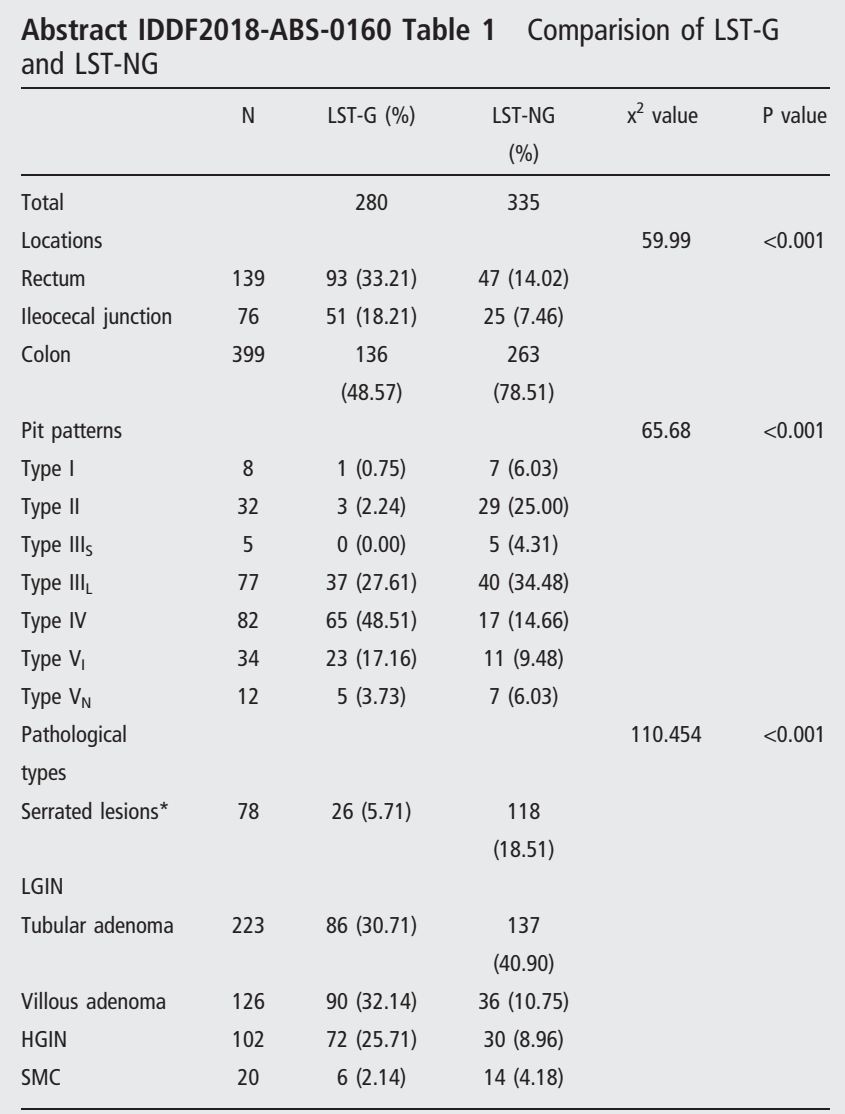

*Serrated lesions: a group of lesions characterised by serrated epithelium, including hyperplastic polyp(HP), traditional serrated adenoma (TSA) and sessile serrated adenoma/polyps (SSA/Ps). LGIN, low grade intraepithelial neoplasia; HGIN, high grade intraepithelial neoplasia; SMC, submucosal carcinoma.

Results A total of 615 colorectal laterally spreading tumours (551patients) were collected. Granular (LST-G) was detected more frequently in the ileocecal region and rectum (75/140, 53.7\%);
Non-granular (LST-NG) were preferentially located in the rest part of the colon. Pit patterns type IV has large proportion in the LST-G and type IIIL in the type LST-NG. On histopathology, LST - NG in the majority with tubular adenoma (40.9\%), while the LST - G than LST - NG more villiform than LST - NG $(32.14 \%$ vs $10.75 \%)$. LST-G and LST-NG had a higher incidence of intramucosal carcinoma and submucosal carcinoma, 27.86\% (78/280) and $13.13 \%$ (44/250), respectively. LST-NG was highest rates of submucosal invasion: $4.18 \%$. To follow-up after treatment 330 cases of patients, 285 cases of lost to follow-up. Follow-up time span of 1 76 months, the median for 15 months. A total of 10 cases of recurrence, endoscopic piecemeal mucosal resection (EPMR) higher recurrence rate was 13.79\% (4/29), compared to nearly 3 years after the ESD treatment, there is no recurrence of LST.

Conclusions Had a higher incidence of intramucosal carcinoma and submucosal carcinoma, in particular, LST-NGPD was highest rates of submucosal invasion, should be noticed by endoscopic physicians. The endoscopic submucosal dissection (ESD) is safe and effective treatment.

\section{IDDF2018-ABS-0164 THE TEMPORAL RELATIONSHIP OF DAILY LIFE STRESS, EMOTIONS AND BOWEL SYMPTOMS IN IRRITABLE BOWEL SYNDROME: A SMARTPHONE-BASED EXPERIENCE SAMPLING STUDY}

${ }^{1}$ Yawen Chan*, ${ }^{1}$ Suzanne Ho-wai So, ${ }^{2}$ Arthur Dun Ping Mak, ${ }^{2}$ Kewin Tien Ho Siah, ${ }^{1}$ Wai Chan, ${ }^{3}$ Justin Che-yuen Wu. 'Department of Psychology, The Chinese University of Hong Kong, Hong Kong; ' Department of Psychiatry, The Chinese University of Hong Kong, Hong Kong; ${ }^{3}$ Department of Medicine and Therapeutics, The Chinese University of Hong Kong, Hong Kong

\subsection{6/gutjnl-2018-IDDFabstracts. 135}

Background The current study aimed to model the moment-tomoment relationship between daily life stress, negative emotions and bowel symptoms among patients with irritable bowel syndrome - diarrhoea subtype (IBS-D) in the flow of daily life using a smartphone-based experience-sampling method (ESM).

Methods Patients with IBS-D $(n=27)$ and healthy controls $(\mathrm{HC})(\mathrm{n}=30)$ completed ESM ratings of their real-time daily life stress, which was defined as the subjective stress related to daily activities, negative emotion and bowel symptoms 8 times a day for 14 consecutive days, following a baseline interview measuring bowel and mood symptoms. Moment-to-moment association between ESM variables was tested within and between groups using multilevel regression modelling.

Results Patients with IBS-D reported more severe bowel symptoms ( $p s<0.01$ ) than HCs, but levels of daily life stress and negative affect were comparable between groups ( $p s>0.05$ ). Time-lagged analysis of ESM data revealed that, among patients with IBS-D, daily life stress predicted a decrease in abdominal pain and urgency to defecation at a subsequent time point ( $p s<0.05$ ), whereas severity of bowel symptoms and occurrence of diarrhoea predicted a subsequent increase in negative affect and daily life stress ( $p s<0.05)$. The above associations were not found among HCs (figure 1).

Conclusions ESM unveiled the dynamic relationship between bowel symptoms, stress and emotionality. Patients with IBS-D responded to bowel symptoms with more stress and distress momentarily. Counter-intuitively, daily life activity stress served as a protective factor for bowel symptoms. Our data informed the psychological understanding of IBS. 